\title{
A Comunicação Científica entre João Jacinto de Magalhães e Antoine-Laurent Lavoisier
}

\author{
ISABEL MARIA MALAQUIAS*
}

\begin{abstract}
Nesta comunicação pretendemos apresentar alguns testemunhos da interacção científica entre João Jacinto de Magalhães e Antoine-Laurent Lavoisier, numa época particularmente rica do desenvolvimento da nova química (1772-1777). A reflexão incide sobre a temática da água e os esforços desenvolvidos para a obtenção de águas minerais artificiais $\mathrm{e}$ dessalinização da água, utilizáveis em viagens maritimas longas, assunto de interesse científico comum.
\end{abstract}

A comemoração do bicentenário da morte de Antoine-Laurent Lavoisier (1743-1794) suscitou-nos a reflexão presente no sentido de o homenagearmos e de desvendarmos também o seu relacionamento com o físico e instrumentalista português João Jacinto de Magalhães (1722-1790).

A física experimental teve, durante o século XVIII, um grande desenvolvimento e o próprio Lavoisier consideravase mais um "physicien" que propriamente um "chimiste", Deve-se a ele, por exemplo, a proposta de criação de uma classe de "physique expérimentale" na Académie Royale des Sciences de Paris[1].

João Jacinto de Magalhães revelou uma actividade variada, tendo-se debruçado sobre temas de física, de química de astronomia, sobre instrumentos $e$ assumido um papel de relevo na comunicação científica da época. Fixou a sua residência em Londres, em fins de 1763 , por esta ser a cidade europeia onde encontrou maior correspondência com os seus interesses de instrumentalista, depois de uma curta estada em Paris, onde integrou, apadrinhado pelo $\mathrm{Dr}$. Ribeiro Sanches, um círculo amplo de homens de ciência e prestígio político social [2, 3, 4a].

Lavoisier entrou, em 1754, para 0 Collège des Quatres Nations (ou Mazarin), tendo, em 1761, abandonado os estudos que o levariam a um bacharelato em Artes, para transitar para 0 de Leis, na perspectiva de seguir os passos paternos. Licenciou-se em 1764, embora 0 seu interesse pela ciência fosse manifesto desde há algum tempo, pois prosseguia a sua formação neste campo, paralelamente. Estudou astronomia com La Caille (1713-1761) e realizou "promenades philosophiques" com o botânico Jussieu, embora esta não fosse uma área para que mostrasse particular vocação. Com La Caille adquiriu inclinação para o quantitativo, bem como interesse nos fenómenos meteorológicos e nas observações barométricas (hipsometria).

0 geólogo Jean-Étienne Guettard, amigo da familia, influenciou Lavoisier a estudar química, pois reconhecendo-Ihe aptidões naturais para a ciência, tê-lo-á aconselhado a frequentar 0 curso de quimica de Rouelle, pois considerava que um mineralogista devia possuir bons conhecimentos de química para melhor identificar e classificar as rochas e minerais. Assim, durante os anos de 1762-63, Lavoisier dedicou-se à Química, enquanto acompanhava Guettard na elaboração de um atlas geológico e mineralógico de França - este era um projecto antigo de Guettard (1746), para o qual teve apoio oficial somente a partir de 1766. Este projecto cartográfico continuou até 1770 , altura em que "completaram e publicaram dezasseis quadrângulos regionais"[1] com simbologia adequada para as diferentes formações rochosas e espécies minerais.

Nas várias saídas que fez com Guettard, Lavoisier recolheu amostras de águas minerais para análise, pois pretendia saber qual o grau de potabilidade das mesmas, ao mesmo tempo que se interrogava sobre as mudanças que teriam ocorrido na Terra e alterado a sua superfície. Isto conduzi-lo-ia a uma teoria da formação das rochas, com base nas de Guettard, Rouelle e Buffon, em que admitiu pela primeira vez, que poderia ter havido uma "sucessão de épocas marcadas por um avanço e retrocesso cíclicos do marn[1], que justificariam a existência, numa região de "terra nova", e camadas litorais (de seixos), formadas em alturas diferentes.
A investigação química mais antiga de Lavoisier remonta ao Outono de 1764. Dizia respeito à constituição do "gypsum", isto é, do gesso. Pretendia Lavoisier que efosse o primeiro artigo de uma série dedicada à análise mineral das substâncias". Este inventário sistemático era para ser efectuado, não pelo método de J. H. Pott - que expunha os minerais à acção do fogo - mas por reacções em solução, por "via húmida". "Procurei copiar a natureza", escreveu Lavoisier. «A água, este solvente quase universal... é 0 agente principal que ela emprega; e também aquele que adoptei no meu trabalho" [1].

A comunicação dos resultados foi feita na Académie des Sciences a 25 de Fevereiro de 1765, tendo sido impressa três anos depois. Nesta altura, Lavoisier aspirava a ser seu membro, 0 que veio a acontecer apenas em 1768; contudo, os seus apoiantes incluíram o seu nome na lista de candidatos em 1766, após este ter concorrido ao prémio da Académie para 0 melhor método de iluminação das ruas de Paris. A intenção era que fosse admitido como "adjoint chimiste", pois vagara este lugar.

Lavoisier continuou os seus trabIhos analíticos que 0 conduziram, em 1768 , a um artigo extenso sobre as suas amostras de água, em que usava como instrumento de precisão o hidrómetro Este artigo foi também lido na Académie e, pouco depois, foi eleito membro (18 de Maio de 1768) juntamente com Antoine Gabriel Jars (1732-1769). Este, que era engenheiro de minas, foi designado "adjoint chimiste" e Lavoisier "adjoint chimiste surnuméraire".

$\mathrm{Na}$ análise das águas minerais, Lavoisier propunha um método diferente do usado habitualmente. Neste fazia-se a evaporação à secura da água e determinava-se, qualitativa e quantitativamente, qual o sal dominante numa dada amostra. No novo método, determinava «a concentração do sal característico, fazendo medidas da gravidade específica da amostra de água com o seu hidrómetrow[1]. 
Algumas águas minerais eram usadas em medicina, pois verificara-se que, em alguns casos, facilitavam o processo digestivo, e em outros, aliviavam os padecimentos causados por cálculos biliares e renais.

Assim, desde os finais do século XVII, fizeram-se análises destas águas, e, por se acreditar que era possível reproduzi-las pela adição de determinados sais, na devida proporção, houve quem as tentasse produzir artificialmente. Verificou-se também que algumas possuíam uma quantidade de gás elevada, que parecia diferente do "ar comum" - o chamado "espírito mineral». Por volta de 1750, o químico francês Venel identificava a existência de uma grande quantidade de gás nas águas de Spa, que não distinguiu do "ar comum"[5]. 0 reconhecimento de que este gás era dióxido de carbono, isto é, o "ar fixo" foi feito por Joseph Black, em 1754.

A identificação da composição do «ar comum" foi um assunto que preocupou grandemente os experimentalistas da segunda metade do século XVIII. Esta questão teve relação também com a produção de águas minerais artificiais.

Por volta de 1770 , Priestley começou a interessar-se pela produção de águas gaseificadas para o que, em 1772 , possuía já um método aceitável - num vaso com cal deitava ácido sulfúrico e transferia, por meio de um tubo abdutor. 0 gás libertado para um outro que se encontrava invertido e cheio de água, sobre uma tina [6].

A referência mais antiga ao relacionamento de Priestley com J. J. de Magalhães data de Março de 1771, altura em que este foi convidado de Priestley para assistir às experiências que iria efectuar numa sessão da Royal Society, em Londres [7a].

Magalhães divulgou o método de Priestley por vários amigos e correspondentes no "continente", a pedido do primeiro, pois mantinha já uma correspondência vasta na época.

Magalhães deverá ter tido conhecimento de Lavoisier, através dos amigos que tinha na Académie des Sciences. Nos primeiros tempos da sua expatriação, J. J. de Magalhães permaneceu por largos periodos em Paris, onde se dedicou a estudos de astronomia com Delisle e Messier, como parece saber-se a partir de uma carta de 1759 [8]. La Caille era entâo um astrónomo de renome, em particular pela sua tentativa de medição do arco de meridiano, no Cabo da Boa Esperança, em 1750. É natural que, por volta de 1762-63, Magalhães tivesse tido quaisquer referências relativas ao jovem promissor que era Lavoisier, que fora aluno do mesmo. Mas destes anos, da saída de Portugal até á sua fixação em Inglaterra, sabe-se muito pouco.

De todo o modo, Magalhães surge, em 1769, ligado a Trudaine de Montigny [9], intendente geral de finanças em França. Este contacto foi feito através de Louis-Henri Duchesne (1724-1793), que era secretário do jovem Montigny. Para além dos interesses relacionados com os cargos que ocupava, que 0 obrigavam a conhecer os avanços tecnológicos existentes em outros paises, nomeadamente em Inglaterra, Montigny tinha uma inclinação especial para a química, que considerava de grande utilidade pública. As notícias de que Magalhães enviava de Londres eram particularmente importantes, pois foi por seu intermédio, e também por cartas enviadas inicialmente ao químico Macquer [10], que as experiências da química pneumática inglesas foram divulgadas em França e influíram no surgimento da "revolução química" [7b]. 0 cargo ocupado por Trudaine de Montigny permitia usufruir do privilégio de franquia, pelo que Magalhães usava o seu endereço para Ihe enviar encomendas de instrumentos, livros, etc., bem como para outros amigos, como Ribeiro Sanches, Messier, Bochard de Saron, Lavoisier.

Em 1771, Magalhães foi eleito membro correspondente da Académie Royale des Sciences, tendo contado com 0 apoio de Trudaine, conforme se depreende do que escrevia a Duchesne, a 23.4.1771[11a]:

"Les idées que vous me donnez par Raport à L'Acad. Roy.", marquent bien La bonté de votre Coeur pour moi, \& caracterisent très notam.' La sincereté de l'amitié dont vous m'honorez. II y a quelque tems, que je m'aperçus de pouvoir reussir, peut etre, dans cette ateinte, ayant egard aux diferens amis, qui semblent etre portés à ma faveur dans L'Academie. Même quelques uns me l'avoient fait entendre: mais ayant fait la reflexion, que celà m'apporteroit un degré de consideration, \& d'honeur tout $\mathrm{Sec}$, qui pour un Philosphe dans ma situation, \& de ma façon de penser, me seroit plus à charge, \& non pas d'aucune façon avantageux, Je n'ai jamais eu la moindre envie de l'entreprendre. Pour le present, L'affaire a une apparence tout à fait differente, par Les esperances, que Vous me marquez dans Votre Lettre: \& outre celà, c'est assez que M.' Trudaine l'approuve, pour que je prend? courage \& me jete entierem.' entre Les dispositions \& avis d'un tel ami comme vous. Ainsi vous pouvez compter, que Je ne manquerai d'baucher une petite Memoire, sur un objet, qui me semble interess.' dans La mechanique, \& que Je tacherai de vous envoyer auplutôt: en vous priant d'en corriger La Language, avec quelque ami intime sur lequel vous pouvez compter.”

A intenção era Magalhães ficar correspondente de $M$. Coumière [11b], amigo de Duchesne, o que não veio a acontecer, por um pequeno precalço, ficando como interlocutor Bory, que conhecera quando ainda era cónego regrante em Coimbra.

As primeiras referências que Lavoisier terá tido de Magalhães parecem corresponder ao conhecimento de cartas deste, que foram lidas na Académie Royale des Sciences, na presença do primeiro. Guerlac [7c] informanos que, quando o químico Macquer leu a carta sobre as observações feitas por Priestley, relacionadas com a purificação do ar viciado, pela presença de plantas, e também sobre as propriedades curativas do "ar fixo", no tratamento de certo tipo de cancro, na sessão de 1 de Abril de 1772, Lavoisier estava presente.

As relações de Lavoisier com Magalhães foram amistosas, tanto quanto é visivel pelas cartas enviadas por este último a Lavoisier e à sua «Epouse philosophique" e mesmo pela afirmação de Grimaux [12b]: "un de ses amis que habitait Londres, Magalhaens ou Magellan".

Magalhães enviou a Lavoisier memórias científicas recentes, surgidas em Londres, em que se incluiam as últimas experiências de Priestley, bem como instrumentos para o seu laboratório no Arsenal.

A 5 de Julho de 1772 [13a], Magalhães escreveu a "um dos seus amigos de Paris" (provavelmente Bory), comunicando o método de Priestley de preparar águas minerais artificiais por meio do "ar fixo", que seria útil na prevenção do escorbuto nas grandes viagens por mar. Mencionava, ainda, todas as descobertas feitas até então sobre este assunto, pelo Dr. Macbride, pelo Dr. Brownring e por Priestley, referindo 



Figura 1

que o método deste último tivera a aprovação do "Collège des Medecins de Londres", pelo que o Almirantado consentira em verificar o mesmo numa viagem aos mares austrais.

Dois dias depois [13b], Magalhães escrevia a Trudaine de Montigny, enviando-Ihe também o metodo de Priestley:

"Le papier ci-joint renferme La methode pour faire des eaux acidules \& calybées, que j'ai abregé en françois \& generalisée à la portée de tout le monde, d'après celle du D.' Priestley, dont je eus I'honneur de vous envoyer des copies imprimées par la poste derniere. Je pense avoir detaillée assez nettement ma particuliere methode, un peu diferente de celle de l'Inventeur, pour ne pas avoir besoin d'aucune planche pour que tout le monde La comprendre.

Si vous pensez si avantageusement de cette converse, comme plusieurs le pensent icy [\& je suis du nombre aussi] Je souhaiterois qu'on La fisse imprimer tout de suite chez vous, car ce ne fera pas audelà d'une feuille d'impression, \& qu'on la repandit gratis par toute La france, pour que tous soient à meme de s'en utiliser. mais il faudra pour Lors en faire corriger le françois, sans en alterer le sens: (...) C'est par cette meme raison que j'ai envoiée une autre copie de ce papier à un autre de mes amis de Paris, \& que je ne manquerais de multiplier s'il m'étoit possible dans si peu de tems de Le rependre par toute L'Europe. (..)"

Este método foi publicado em Londres, em 1772, com o título «Directions for Impregnating Water with Fixed Air in order to communicate to it the peculiar Spirit and Virtues of Pyrmont Water». Magalhães sugeria na sua carta a Trudaine a correcção do francês que usara na sua tradução e pedia a sua divulgação.

Foi esta publicação de Priestley que maior curiosidade despertou em França, onde a necessidade de caracterizar as águas minero-medicinais fora requerida pelo Collège de Médecine a todos os médicos do país [5]. Magalhães comunicara anteriormente experiências feitas em Inglaterra sobre 0 "ar fixo", nomeadamente em cartas a Macquer (1771) [10].

A 7 de Julho de 1772 [11c], Magalhães informava Duchesne do sucesso das suas experiências:

"Je vous envoye ici, mon cher \& bon ami, une nouveauté qui me semble fort utile au public. Je crois que vous ne serez pas faché d'en avoir L'idée pour La communiquer àvos amis dans votre pais, oû je crois que cette Lettre vous trouvera deja. Dans la poste derniere j'envoiai deux brochures angloises avec ce detail à M.' Trudaine \& une pour L'Academie Royale, dans Le Samedi je me suis mis à ecrire quelques copies comme celle ci pour envoyer par La poste à differentes amis, mais j'ai encore rendu hier cette operation un peu plus simple que celleci dans cette Lettre. J'en envois par cette meme poste une copie manuscrite de ma derniere methode à Mons.' Trudaine, \& je souhaterois qu'elle fut imprimée, après que le françois en seroit corrigé, \& qu'on la repandit tout partout. (...)"

A questão acima interessou a Trudaine, que escreveu a Lavoisier, a 14 de Julho [14], sobre 0 assunto, pedindoIhe para repetir as experiências. Informava que iria também verificá-las, pois desde há três dias que o seu laboratório de Montigny estava pronto, e estava ansioso por se tornar um discípulo de Lavoisier:

"J'ai I'honneur de vous envoyer, Monsieur, une lettre que je recois de $M$. Magallhaens Anglois catholique correspondant de l'academie, au sujet d'une decouverte, tres importante Sur l'air fixe. j'y joins la petite brochure de M Priestley qui est parfaitement bien faite a ce qu'il m'a paru. j'imagine que vous entendez bien l'anglois. (o que de facto não era verdade; era M." Lavoisier quem fazia as traduções, devendo-se-Ihe, por exemplo, a tradução do «Essai sur le phlogistique", de Kirwan) vous feriez une très bonne oeuvre très utile de faire publier cet ouvrage traduit avec les notes de $M$. Magallhaens. je vous prie aussi de vou- loir bien repeter ces experiences, et d'ajouter vos propres observations. je vais repetter moi même ces experiences. (...) le merite de ces nouvelles decouvertes etant la promptitude j'espere que vous ne tarderez pas a faire paroistre ce petit ouvrage. il n'y aura pas de mal a en prendre un nombre d'exemplaires pour faire distribuer gratis, principalement aux medecins. dans le desir de donner satisfaction à M. Magalhaens j'ay cru ne pouvoir mieux m'adresser qu'a vous je Scais votre exactitude Sur les details de Phisique et de Chimie et je Scais que c'est vous obliger que de vous mettre a portée de faire une chose utile. (...)"

Não há indícios que Lavoisier tenha repetido as experiências, mas leu, quatro dias depois, na Académie, a carta de Magalhães [7d].

Conforme referimos, Lavoisier também se interessou pela análise das águas, embora mais no sentido da sua adequabilidade para o consumo urbano.

Hả, assim, elos comuns entre Magalhães e Lavoisier em torno da água. Magalhães nāo só divulgou as experiências de Priestley sobre este assunto, como contribuiu para 0 aperfeiçoamento de uma máquina de vidro para a produção de águas minero-medicinais gaseificadas [15], com a qual era possivel produzi-las sem grandes gastos, num intervalo de tempo pequeno e em qualquer local (fig, 1).

Para além de ter apresentado as suas alterações à máquina de Nooth [16], para produção de águas artificiais, Magalhães debruçara-se anteriormente sobre a questão da destilação da água do mar e, também, de águas-ardentes.

Sobre as experiências de destilação da água do mar, sabe-se que

"a primeira ideia da Máquina destiladora que será objecto neste escrito, remonta ao ano de 1770. Executaram-se vários modelos diferentes em tamanho pequeno, \& nomeadamente um no mês de Janeiro de 1773;" finalmente, pouco tempo depois, por ordem de $\propto M$. de Boynes, então Ministro da Marinha, a Máquina foi executada em Paris em ponto grande, (...) Esta Máquina foi então submetida a provas múltiplas, sob os olhares de vários Membros da Académie Royale des Sciences, de numerosas pessoas distintas pelos seus conhecimentos \& pela sua categoria \& o sucesso foi completo." - "[1] M. Turgot, então Intendente de Limoges, actualmente Contrôleur-Général des Finances; $M$. 
Trudaine, Conseiller d'Etat, Intendant des Finances; $M$. Montigny; $M$. Macquer; $M$. Leroy; M. Lavoisier; M. Desmarets, \& plusieurs autres."

Esta passagem foi publicada em 1781, anonimamente, no Journal de Physique [17] de Rozier e consta do prefácio de uma obra, extremamente rara de Magalhães, ambas intituladas «Nouvelle Construction d'Alambic pour faire toute sorte de Distillation en Grand avec le plus d'économie dans l'Opération, et le plus d'Avantage dans le Résultat, en deux parties; La première contenant son application à la distillation des Eaux-deVie; et la seconde à la dessalaison de l'Eau de la Mer à bord des Vaisseaux". Esta publicação saiu anónima, mas, 0 exemplar a que fazemoș referência, existente em Leiden, tem manuscrito "par J. H. de Magellan" e "Ex dono authoris" (fig. 2) [4b, 18].

A 4 de Maio de 1772 [11d], em carta a Duchesne, Magalhães referia-se ao assunto dizendo:

"C'est mon cher ami, pour vous annoncer que J'ai Reussi dans Les Experiences pour faire une grande quantité d'eau douce, avec un apparatus fort aisé, de fort peu de volume: \& peu de depense, à bord des vaisseaux. J'e n'ai pas osé me flater de son effet jusqu'à ce que mes experiences m'enconvainquirent. J'aieté plusieurs fois à bord de ditferents vaisseaux, \& particulierement des deux qui sont destinés pour cette expedition de Mess. ${ }^{\prime s}$. Banks \& Solander, pour y observer tout ce qui pouroit etre utile \& particulierement pour ce qui regardoit cet objet: \& toutes les observations que

Figura 2

NOUVELLE

CONSTRUCTION D'ALAMBIC

POUR FAIRE TOUTE SORTE

DE DISTILLATION EN GRAND, arca

LE PLUS D'ECONOMIE DANS LOPERATION,

LI RLUS DAVANTAGE DANS LE RESUL....

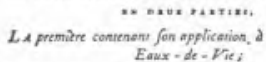

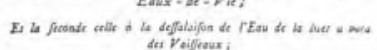

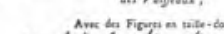
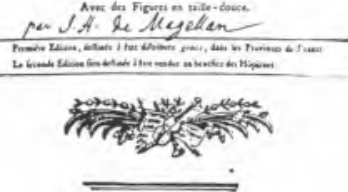

$178 \mathrm{I}$.

ex fows authori j'ai pu faire, jointes à mes tentatives \& experiences, m'ont conduit à deceler cette methode precieuse: dont je vous prie de rendre part à M.' Trudaine pour L'assurer du bon usage que je sais faire du donatif qu'il a eu La bonté de me faire en l'emploiant à des objets avantageux au service du public. Je pense aller toujours à Paris aussitô que Je pourai me degager de mes petits engagements \& arranger quelques petits affaires; (...)".

Na carta de 1 de Junho de 1772 [11e], prosseguia:

"Ayez la bonté mon cher ami, de lire les deux dernieres pages de cette lettre pour M: de Montigny: après celà vous la fermerez \& la lui donnerez de ma part. il s'agit d'arranger les droits sur le eaux de vie: je m'entends sur ces matieres \& puis dire de les connaitre à fond. si M.' Trudaine le souhaite, je n'aurai le moindre dificulté d'aller à Paris, oû je montrerai la methode la plus aisée \& certaine de construire des instruments pour cet objet beaucoup meilleurs que ceux qu'on use en Angleterre dans les douanes: \& dont j'envoie un à $M$ : de Montigny qu'il me demanda: apparament c'est pour la compte de M.' Trudaine. Ia caisse arrivera dans peu jours chez vous (...)"

A existência da versão impressa de Leiden permite esclarecer uma afirmação de Dumas, editor das "0euvres" de Lavoisier - "Cet ouvrage anonyme est l'oeuvre de Lavoisier; il porte son empreinte partout; à son époque, il était seul capable de l'écrire; le principe qu'il pose est le même principe qu'il appliquait bientôt au traitement du salpêtre: obtenir le maximum d'effet avec le minimum de dépense." Grimaux [12b], um dos primeiros biógrafos de Lavoisier, baseado em Dumas, acusa de «abus de confiance" 0 aparecimento da memória no Journal de Physique, que considera ser de Lavoisier.

De facto, Lavoisier realizou também experiências para a dessalinização da água do mar, a pedido de Trudaine, para verificar a utilidade do alambique a que se referia Magalhães. Para isso, Lavoisier mandou e ecutar vários modeIos. Grimaux refere que Turgot [19], que entretanto substituíra Boynes no Ministério da Marinha, decidira a colocação de um alambique num barco do rei, 0 que não veio a acontecer, porque também ele foi substituído.

Tendo terminado as experiências, Lavoisier escreveu uma pequena memó- ria com os resultados, sabendo-se que na "miniatura autógrafa tem: A copier et

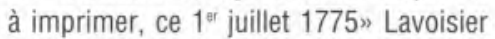
enviou uma cópia a Magalhães.

Magalhães referiu-se algumas vezes às experiências de dessalinização. Na carta a Duchesne, de 27 de Julho de 1773 [11f], considerava:

"Je souhaite bien savoir comment a Reussi M.: Lavoisiere avec la desalution de l'eau Salée. (...)"

Um mês depois [11g], retomava a questão, admitindo que poderia ser útil na concretização das mesmas:

"Je serois bien Charmé de pouvoir faire un petit tour à Paris lors de votre retour. peut etre M. Lavoisiere seroit-il plus á meme de faire aler La machine pour faire l'eau douce de celle de la mer, avec plus de succès si je m'y trouvois $\&^{2}$. Mais je crains ne pouvoir pas me debarasser aussitôt, d'une infinité de petis affaires qui me retient ici (...)"

Mas o seu desejo de ir a Paris, apresentava-se difícil de concretizar, pois, a 2 de Novembro de 1773 [11h], escrevia ainda:

"Je voudrois bien pouvoir faire un tour à Paris dans un ou deux mois d'ici. peut etre pourrions nous alors pousser les experiences de M.' Lavoisiere, qui ne meritent pas de res [este bocado da carta está rasgado] donner cette petite lettre. (...)"

A 11 de Fevereiro de 1774 [13c]. Magalhães escrevia a Lavoisier agradecendo-the 0 envio dos seus "Opuscules de Physique", onde este esclarecia que Venel não era 0 autor da descoberta do "ar fixo", fazendo justiça a Brownring e a Priestley e ultrapassando a questão que Magalhães tivera com Rozier sobre 0 mesmo assunto.

No seu recém-surgido "Journal de Physique», Rozier publicara a tradução do trabalho de Priestley sobre a impregnação das águas com "ar fixo", e estabelecera polémica com Magalhães relativamente às prioridades que Venel teria na identificação das propriedades do referido gás. centava:

Na referida carta, Magalhães acres-

"A present je me flate que vous poursuivez l'objet dela distilation \& desalaison de l'eau de mer par la nouvelle methode; \& je ne doute pas que cette entreprise n'acquiere pas quelques degres de plus grande perfection entre vos mains. Je pense aller à Paris au bout du mois prochain: \& serai très charmé 

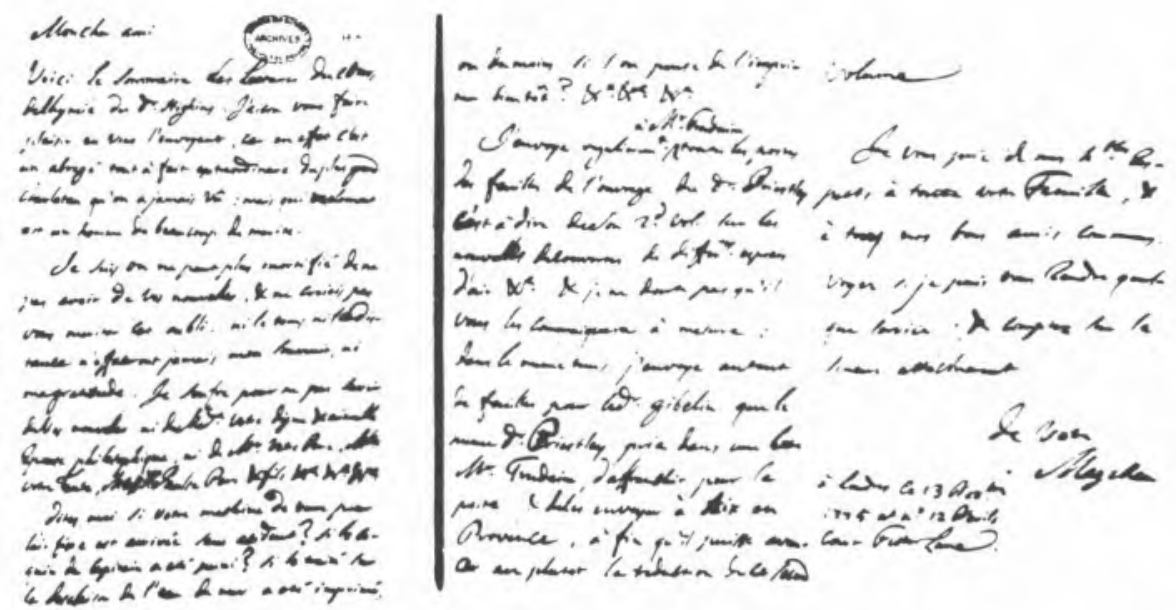

Fig. 3 - Carta autógrafa de Magalhães para Lavoisier

de pouvoir vous aider dans cette entreprise avec les pauvres talents que je possede. (...)"

A 16 de Maio de 1774 [13d], recomendava a Lavoisier, o Dr. Wolfe, de Danzig, que lhe entregaria esta missiva - "un sujet très distingu par son savoir, paritculierem.' dans La Chymie, cette charmant science qui fait vos delices" - e a última publicação de Priestley - 0 1. ${ }^{9}$ volume das "Experiments and Observations on Different Kinds of Air» - Informava-0 também, que na semana seguinte, poderia deslocar-se a Paris.

No mesmo ano, a 24 de Novembro [13c], Magalhães enviava a Lavoisier várias encomendas, via "Mr. Trudaine" $\mathrm{e}$ referia-Ihe 0 seu interesse no método de produzir a cerveja inglesa de que the enviava algumas garrafas. Sobre a dessalinização da água do mar, dizia:

"Vous aurez deja vû dans le voyage du Cap..$^{\text {th }}$ Phips un petit recit ou precis de la maniere employée par Lui \& $M$.' Irwin pour desaler l'eau de la mer: vous y observerez qu'il ne donne pas la figure de la vraie machine à distiller, comme celle que je vous ai communiquée. il paroit qu'on a voulû en cacher encore à la connoissance du Public la vrai construction de cet alambique, dont l'usage est d'une si grande consequence pour toute sorte de distilation en grand. je vous prie de faire cette observation à $M$. Trudaine. j'apporterai avec moi une copie de ce voyage pour sa bibliotheque, $\&$ je vous montrerai ce detour adroit dans la figure qui y est gravée. Je pense partir à la fin de la semaine prochaine, \& de vous embrasser bientôt à Paris. (...)"

Entre a carta acima e a de 13 de Novembro de 1775 ,surge apenas uma, de 14 de Julho deste ano [13f], onde faz referência a encomendas enviadas para Lavoisier que terão sido retardadas por problemas com 0 comandante do navio que as levara.

A 13 de Novembro [13g], perguntava a Lavoisier:

"dites moi si votre machine de verre pour l'air fixe est arrivées sans accident? (...) si le traité sur la desalaison de l'eau de mer a eté imprimé ou du moins si l'on pense de l'imprimer bientôt? (...)

J'envoye regulierem.' à $M$.' Trudaine toutes les postes des feuilles de I'ouvrage du D.' Priestley c'est a dire de son 2." vol. sur les nouvelles decouvertes de differ. ${ }^{\text {tes }}$ especes d'air \& a \& je ne doute pas qu'il ous les communiquera à mesure: (...)" (fig. 3)

A razão para a falta de notícias de Lavoisier, neste último meio ano, devera-se à morte do pai deste, ocorrida entretanto, 0 que Magalhães vem a saber por intermédio de $\mathrm{M}^{\text {me }}$ Lavoisier. Na carta que the dirige a 15 de Dezembro de 1775 [13h], Magalhães referia-Ihe 0 envio de uma brochura que arranjara sobre 0 "modo de fazer o salitre na América" e a continuação das suas diligências para encontrar algumas obras antigas que Lavoisier pedira. 0 assunto do salitre interessava particularmente a Lavoisier, pois além de "Fermier Géneral", desde 1768, pertencia à companhia financeira que administrava a "Régie des poudres et salpêtres".

Magalhães voltava a mencionar a questão do salitre na carta de 16 de Janeiro de 1776 [13i], para referir 0 modo como era obtido na Índia.
Temos conhecimento a seguir de uma carta de 10 de Setembro deste mesmo ano [13j], onde Magalhāes indicava as características da bomba pneumática que remetia a Lavoisier, a qual experimentara em sua casa de 11 de Julho a 20 de Agosto, bem como mais alguns instrumentos e livros para amigos comuns.

Nos «Papiers de Lavoisier» existe ainda um manuscrito de Magalhães, sem data, cujo título é:

«Sur la La Methode pour purifier ou du moins ameliorer l'eau douce dont on fait usage abord des vaisseaux dans des voyages longs. par J. $H$. de Magellan dela S. R. de Londres Corresp. del'Academie".

0 facto de Magalhães se apresentar como membro da Royal Society implica que a data deste manuscrito é posterior a 21 de Abril de 1774 .

Nesta carta descreve um dispositivo de filtração sucessiva da água, que ilustra, a fim de lhe retirar as partículas em suspensão, que estariam na origem do apodrecimento da água (fig. 4).

A carta de 31 de Janeiro de 1777 [13k] não foi escrita por Magalhães (carta ditada ou copiada). 0 seu interlocutor não está explicitado, não sendo Lavoisier, pois se diz "Mon cher Chevalier et très cher ami». Nela descrevem-se as experiências que tinham sido feitas na sua presença com as novas máquinas pneumáticas, de que tinha já enviado uma para Lavoisier, com os aperfeiçoamentos novos introduzidos por Smeaton.

Magalhães pedia para se divulgarem estas experiências na Académie des Sciences, ao mesmo tempo que informava que Lavoisier poderia fazer demonstrações com a sua máquina pneumática,




na Académie. Terminava, indicando que Rozier as poderia publicar no "Journal de Physique", se assim o entendesse.

Não se conhecem cartas posteriores a esta data endereçadas a Lavoisier Deste último, há uma carta de 30 de Abril de 1785 [20] em que Lavoisier recomenda a Magalhães o presidente de Virly, pedindo-Ihe para 0 apresentar nos meios científicos.

Apesar de não existirem, tanto quanto é do nosso conhecimento, mais cartas de Magalhães para Lavoisier, e vice-versa, Magalhães não deixou de se interessar pelos temas que conduziram Lavoisier a um caminho novo para a química. Através de cartas dirigidas a outros amigos, sabe-se que as experiências efectuadas por Lavoisier e seus adeptos, relativas à calcinação dos metais e combustão em geral, continuaram a preocupar João Jacinto de Magalhães nos anos subsequentes e a servir de motivo de debate [21].

\section{AGRADECIMENTOS}

Agradeço ao Prof. Doutor Fernandes Thomaz a leitura crítica e sugestōes oportunas.

\section{NOTAS E REFERENCIAS}

1. C.C., Gillispie: Dictionary of Scientific Biography - Lavoisier, art. ${ }^{\circ}$ de Henry Guerlac, Charles Scribner's Sons, New York, 1981.

2. Maximiano de Lemos: Estudos de História da Medicina Peninsular, Porto, 1916.

3. Joaquim de, Carvalho Correspondência cientifica dirigida a João Jacinto de Magalhães, Revista da Faculdade de Ciências, Universidade de Coimbra, 20 (1951) 93-283.

4a. Manuel FernandesThomaz, Isabel Maria Malaquias: Joāo Jacinto de Magalhães: a sua obra impressa e a sua correspondência científica, Revista da Universidade de Aveiro / Letras, n.* 45. (1987/88) 7-56.

4b. idem, p. 23.

5. Noel G. Cole: The preparation and uses of artificial mineral waters (ca. 1680-1825), Ambix, 31 Part I (1984) p. 32-48.

6. Joseph, Priestley: Directions for Impregnating Water with Fixed Air in order to communicate to it the peculiar Spirit and Virtues of Pyrmont Water. London, 1772.

7a. Henry Guerlac, Lavoisier - The Crucial Year, Cornell University Press, Ithaca, New York, 1961 , p. 51 - retirado do Journal Book da Royal Society.

7b. idem, p. 36-75

7c. idem, p. 55.

7d. idem, p. 58-9 - Procès-verbaux, 91 (1772), fol. 254 verso.
8. Rómulo de Carvalho: "Quatro cartas inéditas de João Jacinto de Magalhães", comunicação na « João Jacinto de Magalhães Conference», Coimbra (1990) (para publicação nas Actas).

9. Jean-Charles-Philibert Trudaine de Montigny (1733-1777) era filho de Daniel Charles Trudaine (1703-1769), conhecido por "o grande Trudaine», que foi Conselheiro de Estado. Intendente Geral de Finanças e Director da Administração de Pontes e Estradas. Trudaine de Montigny, referido muitas vezes como «Mr. Trudaine», foi preparado pelo pai para the suceder. Teve um interesse mais profundo pela ciências e tecnologia do que o pai e maior do que as funçōes públicas que veio a exercer, requeriam que tivesse. A actividade pública de ambos foi notável.

Em 1766, pretendeu que a Académie instituisse um prémio de 1200 libras, que disponibilizaria, com vista à distinção do melhor método de fazer vidro "flint", indispensável na construção de lentes acromáticas 0 mesmo veio a ser atribuido por Luis XV; dada a importância do assunto. Outra contribuição importante de Trudaine, para a Académie, foi a aquisição de uma gigantesca "lente de queimars, por 15000 libras, conhecida por «lente de Trudaine». Esta lente foi usada por Lavoisier, e outros, em 1774.

- v.d. artigo de Ralph E. Oesper: «Priestley, Lavoisier, and Trudaine de Montigny», Journal of Chemical Education, 13 (1936) 403-412.

10. Cartas de Magalhães para Macquer de $4.5 .1771,20.8 .1771,5.10 .1771$ e 25.10 .1771 . Bibliotèque Nationale, Paris, Ms. Fr. 12306.

11a. Archives Nationales, carton $T 160^{10-12}$, carta de 23.4.1771 para Duchesne.

11b. idem, idem, carta de 1.10.1771 - «Vous aurez deja lû que mon election en correspondent del'Academie est deja faite: \& je suis bien faché d'avoir manqué en partie notre bût: qui etoit d'avoir $M$ : Coumiere pour Recevoir ma correspondence (...)

11c. idem, idem, carta de 7.7.1772.

11d. idem, idem, carta de 4.5.1772.

$11 \mathrm{e}$. idem, idem, carta de 1.6 .1772

11f. idem, idem, carta de 27.7.1773.

11g. idem, idem, carta de 27.8.1773.

11h. idem, idem, carta de 2.11.1773.

12a. Édouard Grimaux: Lavoisier (1743-1794) d'après sa correspondance, ses manuscrits, ses papiers de famille et d'autres documents inédits, deuxième édition, reimpressão pelas Éditions Jacques Gabay, 1992, p. 51 .

12b. idem, p. 137-138

13a. Archives Nationales, "Papiers de Lavoisier", Ms 1642 A, carta de 5.7.1772.

13b. idem, idem. Ms 1642 B, carta de 7.7.1772. carta para Trudaine de Montigny.

13c. idem, idem, Ms $1642 \mathrm{D}$, carta de 16.5.1774.

13d. idem, idem, Ms $1642 \mathrm{E}$, carta de 16.5.1774

13e. idem, idem, Ms 1642 F, carta de 24.11.1774.
* Departamento de Física

Universidade de Aveiro

13g. idem, idem, Ms $1642 \mathrm{H}$, carta de 13.11.1775.

13h. idem, idem, Ms 1642 I, carta de 15.12.1775.

13i. idem, idem, Ms $1642 \mathrm{~K}$, carta de 16.1.1776.

13j. idem, idem, Ms $1642 \mathrm{~L}$, carta de 10.9.1776.

13k. idem, idem, Ms 1642, carta de 31.1.1777.

14. René Fric (ed.): Oeuvres de Lavoisier Correspondance, Tome II (1770-1775), Paris 1957, p. 368-369. Carta de 14.4.1772 de Trudaine para Lavoisier. A mesma carta está publicada em H., Guerlac: op. cit. nota 7a, p. 57-58.

15. J. H. Magellan: Description of a GlassApparatus for Making in a few Minutes, and at a very small Expence, the Best Mineral Waters of Pyrmont, Spa, Seltzer, Seydschutz, Aix-la Chapelle, \&c. together with the Description of two New Eudiometers, .... and the Method of using these Instruments, in a Letter to the Rev. Dr. Priestley the third edition, London, MDCCLXXXIII

16. Após Magalhães ter divulgado o método de Priestley para produção de águas minerais artificiais, o construtor de instrumentos Blunt concebeu uma máquina para 0 efeito, tendo John Merwin Nooth, M.D., idealizado outra, menos perfeita, posteriormente, que Parker aperfeiçoou.

17. Journal de Physique (1781), Tome XVIII, Par. II, Juillet, p. 3-19+1 Planche; Aout, p. 85-103+3 Planches.

18. David Willemse: "Suites d'un voyage aux Pays-Bas - John Hyacinthe de Magellan et ses rapports avec J. H. van Swinden", Arquivo do Centro Cultural Português de Paris da Fundação Calouste Gulbenkian, volume VII, p. 225-278 (1973)

19. Anne-Robert-Jacques Turgot (1727-1781) fo ministro de Estado, "controlleur général des Finances" e ministro da Marinha. Caracterizou-se pelo seu saber enciclopédico e apoio às ciências. Aconselhava-se frequentemente com cientistas nomeadamente com Condorcet; patrocinou traducões de obras cientificas e encorajou o trabalho dos inventores. Contribuiu para a "Enciclopédie" de Diderot e d'Alembert com um artigo intitulado "Expansibilité».

A memória de Magalhães, sobre os octantes e sextantes ingleses, que foi impressa sob Privilégio da Académie Royale des Sciences, fo por este dedicada a Turgot.

20. Joaquim de Carvalho, op. cit. na nota $3, p$. 253, refere-a como sendo de 1789, embora indique que esta surgiu, com data de 1785 , no Catalogue préliminaire de la Correspondance de Lavoisier, elaborado por René Fric (1949). Esta carta pertence à Oxford Bodleian Library, Ms. Riguad 38, fol. 116. Na ediçăo de M. Goupil dos Fasc. IV das Oeuvres de Lavoisier - Corres pondance (1784-1786), de 1986, a carta é referida como sendo de 1785 .

21. Isabel M. Malaquias, Manuel F. Thomaz, John Hyacinth de Magellan and the Phlogiston Theory, comunicação na "João Jacinto de Magalhães Conference», Coimbra (1990) (para publicação nas Actas). 